\title{
ETIOLOGIA DA TENSÃO PRE-MENSTRUAL: REVISÃO DA LITERATURA
}

\author{
Rui Miranda *
}

A tensão pré-menstrual (TPM), referida pela primeira vez há pouco mais de trinta anos, já foi objeto de mais de uma centena de publicacōes, sendo controversa a sua etiologia. Revisão da literatura permite apresentar diversas hipóteses etiológicas na seguinte ordem: fatôres endócrinos, fatôres psicológicos, fatôres psicológicos e endócrinos, alergia, toxemia, avitaminose.

Fatôres endócrinos - Como conseqüência do aumento pré-menstrual dos estrógenos circulantes, ocorrem múltiplos efeitos, interessando-nos particularmente aquêles citados por Morton e col. ${ }^{30}$ : maior proliferaçãc epitelial das mamas e dos órgãos pélvicos, alteração do metabolismo dos eletrólitos e da água com retenção de fluído extracelular (edema) e alterações do metabolismo dos carboidratos com queda do nível sangüineo da glicose.

Os que atribuiam à hiperestrogenemia os diversos sinais e sintomas da TPM podem ser enquadrados em duas correntes: para a primeira, o aumento de estrógenos é absoluto, e para a segunda, relativo. A hipótese da hiperestrogenemia absoluta foi levantada por Frank ${ }^{17}$ admitindo que um limiar elevado de excreção renal dos estrógenos seria a causa primária; a hiperestrogenemia, por sua vez, levaria ao edema pré-menstrual que, ao lado de uma "sensibilidade individual" aos estrógenos, determinaria o aparecimento da TPM. Para Albeaux-Fernet e col.1, além do edema, teriam importância os estímulos trófico e secretor dos estrógenos sôbre os órgãns femininos e sôbre as glândulas. Entretanto, Israel ${ }^{25}$ verificou que o limiar đe excreção renal para os estrógenos estava elevado em apenas alguns casos de TPM, o que punha em dúvida a hipótese de Frank. Israel verificou a elevada incidência de ciclos anovulatórios no seu grupo de estudo e, baseado nessas observações, levantou a hipótese de que a TPM fôsse decorrente, não de um aumento absoluto de estrógenos circulantes, mas de um aume: $:$ to relativo, em virtude de luteinização deficiente, com baixa na produção de progesterona; entretanto, assinalou que tal hipótese era ameaçada pela ausência de TPM em algumas mulheres com ciclos anovulatórios. Morton $* 9$ incluiu-se entre os que acreditam na influência do aumento relativo dos estrógenos e chamou a atenção para a hipoglicemia subclínica que então ocorre e que poderia ser responsável por algumas das queixas pré-menstruais. Shick ${ }^{41}$, baseado na ocorrência de depressões pós-parto, atribuiveis à súbita queda dos niveis de progesterona, admitiu que a deficiente produção pré-

\footnotetext{
* Instrutor de Ensino no Departamento de Neuro-Psiquiatria-Psicologia, setor de
} de Psicologia, da Faculdade de Medicina da Universidade de Minas Gerais. 
menstrual dêste hormônio seja responsável pelas depressões que tão frequentemente ocorrem neste periodo.

Gillman ${ }^{19}$ verificou que a administração de progesterona na primeira metade do ciclo reproduzia a TPM típica em $7 \%$ ou reações similares em $43 \%$ dos casos, observando que algumas mulheres eram profundamente afetadas por pequenas doses, enquanto que outras, mesmo após grandes doses, nada sentiam. Tendo por base tais observações Gillman admitiu que a progesterona seria, ou não, responsável pela sindrome, dependendo apenas da sensibilidade do sistema nervoso a êste hormônio. Para Pellanda ${ }^{33}$ a TPM seria determinada pelo acúmulo de água no periodo pré-menstrual; a retenção de água não seria devida aos estrógenos diretamente, mas sim à existência de maior quantidade de mineralocorticóides, em relação aos estrógenos.

Robinson e Farr ${ }^{38}$ assinalaram um efeito antidiurético moderado em pacientes com edema pré-menstrual, o que não ocorria no periodo em que não havia edema, o que os levou a atribuir a TPM ao aumento do hormônio antidiurético. Bickers e Woods ${ }^{7}$ relataram a reprodução parcial da síndrome após administração de pitressina.

Os bons resultados obtidos por Day ${ }^{13}$ no tratamento da TPM com estrógenos, levaram-na a admitir que a síndrome é determinada pela queda da produção dêste hormônio (hipoestrogenismo).

Fatôres psicológicos - São múltiplas as causas psicológicas invocadas para explicar a TPM. Citaremos as principais, ressaltando que algumas constituiram apenas motivo de especulações enquanto que outras foram suficientemente demonstradas: tensões familiares aumentadas por relacionamento pouco maduro (Fortin e col.16, Fortin \& Wittkower ${ }^{15}$, Paulson ${ }^{32}$ ); atitude de mêdo em relação à menarca e menstruações, decorrente de mau preparo pela mãe (Brush ${ }^{9}$, Fortin e col.16, Fortin \& Wittkower ${ }^{15}$, Paulson ${ }^{32}$, Shainess ${ }^{40}$ ); tendência a perceber o fluxo menstrual como experiência pessoal traumática e dolorosa (Paulson ${ }^{32}$ ); percepção do fluxo como uma experiência que dificulta a execução dos papéis sociais femininos (Paulson ${ }^{32}$ ); situações traumatizantes tais como afastamento do lar, separação dos pais, sentimento de culpa acêrca de tentações sexuais (Fortin e col. ${ }^{16}$, Fortin \& Wittkower 15); repúdio do papel feminino com marcado sentimento de inveja do homem (Fortin e col.16); mêdo da gravidez (Cooke ${ }^{12}$ ); sentimento de culpa pelas mudanças que ocorrem nos órgãos genitais (Horney 24); horror à menstruação, reprimido desde a época do homem primitivo (Chadwick ${ }^{11}$ ).

Rogers ${ }^{39}$, baseado no trabalho de Lamson e col. ${ }^{28}$ no qual ficou demonstrada uma relação entre o grau de tensão nervosa e a produção de aldosterona, admitiu que fatôres psicológicos não apenas seriam responsáveis pelas queixas psíquicas da TPM, mas também pelo edenıa (com os sinais e sintomas físicos dêle resultantes), via hiperaldosteronismo.

Fatôres psicológicos e endócrinos - Alguns autores acreditam nos fatôres psicológicos sem desprezar as alterações endócrinas, enquanto que outros admitem que os dois tipos de fatôres são inseparáveis. 
Para Benedek \& Rubenstein ${ }^{5}$ os estrógenos são responsáveis pelos impulsos heterossexuais da mulher, máximos na ovulação, quando são mais elevados os niveis dêstes hormônios; após esta fase a tensão sexual diminul, com retôrno a um estado mais passivo-receptivo, com marcado desejo de ser amada, de receber gratificação ou de ser cuidada, com maior interêsse peìo próprio corpo e com fantasias ocasionais da gravidez; tais atitudes foram associadas à elaboração de progesterona. Assim, os hormônios ovarianos seriam responsáveis pelas expressões instintivas, inconscientes, dependendo da produção normal ou anormal de estrógenos ou de progesterona ou da quantidade relativa dêles;. quando houvesse anormalidade nessa produção a TPM poderia instalar-se.

Taylor ${ }^{43}$ expressou a opinião de que os fatôres psíquicos sejam freqüentemente responsáveis pelo excessivo ingurgitamento vascular das estruturas pélvicas, originando a "síndrome de congestão pélvica crônica". A dor e o desconfôrto decorrentes dessa congestão muito contribuiriam para o aparecimento de angústia e depressão crônicas, comuns nessas mulheres. No periodo pré-menstrual quando, por influência hormonal, essa congestão é máxima, tais sintomas se acentuariam e a TPM se estabeleceria.

Rees ${ }^{36}$, baseado nas experiências de Selye determinando a reação de alarme da síndrome de adaptação geral mediante a administração de estrógenos, admitiu que o mesmo poderia ocorrer com o aumento relativo dos estrógenos no período pré-menstrual, além das alterações físicas decorrentes do desequilíbrio hormonal. Por outro lado, os fatôres psicológicos também alimentariam a reação de alarme. A tudo isso seria somada a presença de neurose, o tipo de personalidade, o grau de estabilidade emocional. O resultado seria a TPM.

Para Torghele ${ }^{45}$, as alterações orgânicas da segunda fase do ciclo ovariano desencadeariam um "stress" que, por sua vez, ativaria os conflitos emocionais conduzindo à TPM.

Alergia - Alguns autores defendem a teoria de que as mulheres portadoras de TPM seriam sensiveis aos próprios hormônios ovarianos; baseiam-se no encontro de eosinofilia pré-menstrual e de reações cutâneas a êstes hormônios, em muitas mulheres portadoras da síndrome (Riebel ${ }^{3 i}$, Zondek \& Bromberg 47, Amaral 2, Cazzola ${ }^{10}$, Mukherjee ${ }^{31}$, Arvay \& Myiri ${ }^{4}$ ).

Toxemia - Para outros, o importante é a "toxemia aquosa" como determinante dos sintomas; o edema cerebral seria o responsável pelos sintomas psicológicos; o quadro seria tanto mais dramático quanto maior fôsse a retenção aquosa, assumindo proporções severas quando o aumento do pêso excedesse $2 \mathrm{~kg}$; o fator primário poderia ser hormonal ou tóxico (toxinas metabólicas de aparecimento cíclico, tal como a toxina de Macht ou menotoxina) ou mesmo ambos (Stieglitz \& Kimble ${ }^{42}$, Bickers ${ }^{6}$, Eichner \& Waltner ${ }^{14}$ ). Krasowska ${ }^{26}$ admitiu que um fator tóxico resultante das ações dos estrógenos, atuando sôbre o aparelho visual, levaria ao aparecimento das alucinações visuais das psicoses pré-menstruais. 
Avitaminose - Biskind ${ }^{8}$ admite que a deficiência de vitaminas, particularmente as do complexo $\mathrm{B}$, dificultando a inativação dos estrógenos pelo fígado, conduziria ao acúmulo dêstes no sangue e daí surgiria a TPM.

Assinalamos a existência de 10 correntes etiológicas que tentam explicar a TPM e verificamos que dentro delas existem algumas divergências. Isto espelha o quanto é controvertido e discutido o assunto e, por essa razão, não nos surpreenderemos se existirem outras teorias além das que foram citadas, sendo provável que novas hipóteses surjam antes que o problema seja resolvido. O estudo da TPM em geral e de sua etiologia em particular é difícil em face dos múltiplos fatôres em jôgo: de um lado os hormônios e, em especial, os ovarianos; de outro, o comportamento; entre os dois, os pontos de contacto.

Analisaremos as 10 correntes etiológicas separadamente, podendo dizer desde já que, entre as hipóteses citadas, 4, isto é, as que tentam explicar a TPM pelo hipoestrogenismo, pela sensibilidade individual à progesterona, pela avitaminose e pelo aumento do hormônio antidiurético não têm muita aceitação.

Quanto à hiperestrogenemia, Greenblatt 21 lançou mão dos andrógenos, com bons resultados, em dois casos de TPM, partindo do princípio de que o antagonismo dos estrógenos em excesso deveria eliminar os sintomas; por outro lado, Mukherjee ${ }^{31}$ usou essa mesma terapêutica num grupo de pacientes com TPM, obtendo algumas melhoras em graus variáveis mas assinalando que, em algumas pacientes, os sintomas agravaram-se. Com resreito à hipótese da hiperestrogenemia relativa em virtude da deficiente produção de progesterona, os resultados obtidos com a sua administração são bcns, mas não são ideais (Israel ${ }^{25}$, Mukherjee ${ }^{31}$, Appleby ${ }^{3}$, Greene \& Dalton ${ }^{22}$ ). Além da observação de Israel ${ }^{25}$ no sentido de que muitas mulheres com ciclos anovulatórios não têm TPM, devemos acrescentar que a sindrome tem incidência maior do que os ciclos anovulatórios. Se fôssemos aceitar a hipótese da luteinização deficiente, tôdas as mulheres com ciclos anovulatórios deveriam ter TPM; como isso não ocorre, é menos provável que êste mecanismo possa ser o único responsável pela síndrome, principalmente naquelas mulheres que, apesar de ovularem, têm graus menores de luteinização deficiente. Assim sendo, as evidências impõem dúvidas quanto à importância dada ao aumento dos estrógenos.

A propósito da ação da progesterona associada ̀̀ sensibilidade individual, não são concludentes os resultados de Gillman ${ }^{19}$ administrando progesterona na primeira fase do ciclo e reproduzindo a síndrome em graus variáveis. Podemos supor que êste hormônio produziu, nas mulheres estudadas, uma retenção de água, isto é, uma modificação no organismo que deu lugar ao aparecimento de alterações no comportamento de algumas delas, em virtude de uma certa labilidade, não do sistema nervoso como quís o autor, mas de sua estrutura psicológica. Podemos admitir que nas mulheres bem estruturadas e que tinham maiores facilidades em aceitar suas alteraçōes fisiológicas, ocorreram apenas alguns sinais e sintomas físicos, sem haver comprometimento da arquitetura psicológica. Dai esperarmos que a progeste- 
rona tenha determinado o aparecimento da TPM nessa experiência, não por efeito direto, mas sim indiretamente, tal como poderia ter ocorrido se o autor tivesse usado estrógenos ou hormônio antidiurético ou mineralocorticóides. Seria, pois, necessário algo mais que progesterona; isto é, seria necessário mais alguma coisa além da retenção aquosa; e isto poderia ser a labilidade da arquitetura psicológica às alterações do organismo.

A propósito da ação dos estrógenos e função cortico-supra-renal, convém assinalar que Bickers \& Woods $^{7}$ afirmavam que os corticóides supra-renais estão elevados no periodo pré-menstrual e que Mukherjee 31 encontrou pequeno aumento dos 17-cetosteróides urinários nesta época, sem contudo poder estabelecer relação com o aumento e intensidade do edema. Por outro lado, outros autores (Thorn e col.44, Morton ${ }^{29}$, Fortin e Wittkower ${ }^{15}$ ) mostraram que nem tôdas as mulheres portadoras de TPM têm aumento de pêso no periodo pré-menstrual e que o aumento ocorre também em mulheres que não apresentam queixas de TPM; Bickers \& Woods i afirmaram que a sintomatologia é proporcional a êste aumento, sendo severa quando êle é superior a $2,5 \mathrm{~kg}$. Bàsicamente, determinando as queixas, existe o edema para os que crêem que no aumento da função cortico-supra-renal está a etiologia da síndrome. $\mathrm{E}$ neste caso, o raciocínio anterior é aplicado aqui também.

A literatura não nos dá informação seguras a respeito do aumento do hormônio antidiurético. $\mathrm{O}$ fato de Bickers \& Woods $^{7}$ haverem reproduzido a síndrome mediante a administração de pitressina não significa que êste hormônio esteja aumentado no período pré-menstrual. Caso isso ocorresse, o edema, bem como alguns sinais e sintomas físicos, seriam explicados, mas não a síndrome total.

Faltam-nos maiores informações sôbre a hipótese do hipoestrogenismo. Freed \& Kroger ${ }^{18}$ admitiram que o estrógeno usado por Day ${ }^{13}$ pudesse ter suprimido a ovulação, o que teria conduzido a uma interpretação errônea dos resultados.

No que respeita aos fatôres psicológicos, Veit 46 afirmou que os conflitos invocados para explicar a TPM podem estar presentes em mulheres que nada sentem no período pré-menstrual; além disso, êste autor manifestou-se contra a atitude de "inferir de certos conflitos inconscientes uma doença inteira". Estudando um grupo de adolescentes e moças, Golub \& Menduke ${ }^{20}$ não encontraram relação entre a TPM e o ajustamento social. Israel ${ }^{25}$ afirmou que a psicoterapia é ineficiente. Por outro lado, Henriksen ${ }^{23}$ admitiu que a psicoterapia, visando a ajudar a paciente a compreender melhor a si mesma e aos seus problemas, é inestimável; Fortin e col.j6 afirmaram que bom número de pacientes respondeu à psicoterapia sem o emprêgo de medicação combinada. Rees ${ }^{36}$ observou relação direta entre constituição e predisposição à neurose e a incidência da TPM, relação direta entre a severidade da síndrome e da neurose, maior desajustamento no grupo portador de TPM e, além disso, que muitas pacientes neuróticas responderam à terapêutica psiquiátrica sem melhora significante da TPM; observou ainda que a TPM pode ocorrer em mulheres com pouca ou nenhuma evidência de ou predisposição para a neurose e que há muitas mulheres 
severamente neuróticas sem TPM significante. Lamb e col. ${ }^{2 i}$ fizeram estudos eletrencefalográficos, hormonais e psiquiátricos em 5 moças portadoras de TPM e 5 moças contrôles, não encontrando diferenças essenciais por êste método; verificaram, entretanto, diferenças no comportamnto das componentes dos dois grupos, mesmo fora do período pré-menstrual, caracterizado por maior "labilidade" das portadoras de TPM.

Os autores que fazem referência à psicoterapia, no material que nos foi possivel compulsar, não relatam observaçōes meticulosas, não havendo dados estatísticos nem descrições das técnicas usadas. Com respeito aos interessantes achados de Rees ${ }^{36}$, ficamos insatisfeitos pois nenhuma informação foi dada com respeito à psicodinâmica das pacientes neuróticas sem TPM e das mulheres tidas como normais e que apresentavam a sindrome.

A observação de Veit 46 de que os conflitos invocados como causadores da TPM são encontrados em mulheres que nada sentem no período prémenstrual, faz pensaŕ que êles sejam insuficientes para determinar o aparecimento dos sintomas a não ser que, em tais mulheres, o grau de aceitação das mudanças fisiológicas seja grande; mas neste caso deixaríamos de falar em "conflitos". O mais importante, talvez, não seja buscarmos os "conflitos causadores da TPM" mediante questionários prèviámente elaborados, mas considerarmos cada caso individualmente, procurando conhecer sua psicodinâmica.

A idéia de Rogers ${ }^{39}$ com respeito à possibilidade da psicogênese de todos os sinais e sintomas da TPM é muito atraente mas carece de confirmação experimental. Temos de aceitar as dificuldades naturais dêste terreno e admitir que a hipótese da psicogênese da TPM ainda deverá ser muito explorada antes de ser destruida ou firmada.

Quanto à associação de fatôres psicológicos e endócrinos, os achados de Benedek \& Rubenstein ${ }^{5}$ deixam-nos na expectativa de que dramáticas alterações ocorreriam na gravidez e na menopausa de mulheres portadoras de TPM; mas isso nem sempre se dá. As idẽias de Rees 36 são muito interessantes quando êle admite que alterações hormonais determinariam sinais e sintomas da TPM de um lado e que, de outro, seriam igualmente importantes os problemas ambientais, a existência ou predisposição para neurose, o grau de estabilidade geral, o tipo de personalidade, as atitudes para com o ciclo menstrual. Para Torghele ${ }^{45}$, além da participação hormonal, temos de considerar os conflitos, que não são especificos como querem alguns autores, mas variam de pessoa para pessoa e de situação para situação.

Para Mukherjee ${ }^{31}$ a alergia deve ser levada em conta em alguns casos. As pesquisas nesse campo são poucas e a experiência existente é mais no sentido do tratamento com anti-histamínicos e dessensibilização hormonal, sendo que cś resultados foram considerados bons pela maioria dos experimentadores que defendem esta teoria.

Quanto à toxemia, consideramos como razoável a explicação de vários sinais e sintomas pela ação da "toxemia aquosa"; contudo, as queixas psíquicas são mais difìcilmente explicáveis; o possivel edema cerebral determina sintomas mais específicos (dor de cabeça, crises convulsivas) do que os sintomas psíquicos da TPM. Devemos admitir, contudo, que as altera- 
ções físicas de uma retenção aquosa possam contribuir para desencadear ou exacerbar conflitos que envolvem as esferas menstrual e genital, problemas neuróticos atuais ou em potencial e problemas situacionais, como querem Rees ${ }^{36}$ e Torghele ${ }^{46}$. Com respeito às toxinas, falta-nos evidência do papel desempenhado por elas na sindrome.

Além das informações prestadas por Biskind ${ }^{8}$, a literatura não nos fornece estudos que neguem on confirmem a hipótese etiológica que incrimina a avitaminose. De qualquer forma ela defende, em última análise, um aumento de estrógenos circulantes e, neste caso, cairíamos na discussão da hipótese inicial.

As medidas terapêuticas são tomadas com base nessas teorias. Conduta muito seguida na prática é a eclética: se existem sinais evidentes de retenção de água, lança-se mão de dieta sem sal e de diuréticos; se os resultados são parciais e os sintomas psicológicos são muito evidentes, associase uma droga de ação central (meprobamato, derivados fenotiazínicos, antidepressivos); se as queixas persistem total ou parcialmente, usa-se a progesterona; a pesquisa da alergia é indicada quando falham as medidas anteriores. O tratamento é feito nos dias das queixas da TPM. Numerosas combinações são feitas, até encontrar-se o ideal, ou o que dele se aproxime, -para cada paciente; tais combinações são comuns nos produtos comerciais específicos. Com respeito aos placebos, êles não produziram melhoras significantes (Morton e col. ${ }^{30}$, Appleby ${ }^{3}$, Pünder ${ }^{35}$ ).

Com relação aos diuréticos, Pellanda 33 verificou que os mercuriais dão bons resultados apenas no primeiro mês e podem levar até à oligúria paradoxal nos seguintes; Bickers \& Woods ${ }^{\top}$ verificaram que os derivados da teofilina, além de darem ótimos resultados, bloqueiam os efeitos da pitressina administrada.

A escolha da droga para combater as queixas psicológicas dependerá do quadro. Bons resultados com meprobamato, em doses variáveis em função da intensidade das queixas, foram obtidos por Pennington ${ }^{34}$ e Appleby ${ }^{3}$.

A progesterona foi usada na dose de $10 \mathrm{mg}$ por via intramuscular em dias alternados (Mukherjee ${ }^{31}$ ) e na de $25 \mathrm{mg}$ (Greene e Dalton ${ }^{22}$ ).

A psicoterapia deve ser sempre considerada e deve ficar a cargo de psicoterapeuta. Ela não deve estar voltada para a TPM apenas, mas para o indivíduo globalmente, visando a reestruturar as percepções acêrca de si próprio e do mundo em que vive, com o que êle adquire compreensão progressivamente maior de si (inclusive das suas modificaçōes fisiológicas) e do mundo; a compreènsão abre caminho para a aceitação. As alterações prémenstruais tendem então a ser vistas sob nôvo ângulo, deixando de constituir motivo de "tensão".

\section{RESUMO}

O autor faz uma revisão da literatura sôbre a etiologia da tensão prémenstrual, distinguindo 10 correntes que são esquematizadas e discutidas; verifica que nenhuma delas oferece base suficiente para explicar a sindrome 
de forma convincente. Com respeito à psicogênese, expressa a opinião de que dêste campo ainda não foi suficientemente explorado.

\section{SUMMARY}

\section{Etiology of premenstrual tension: review of the literature}

The author reviews the literature regarding the etiology of the premenstrual tension, considering 10 distinct currents which are discussed. He finds no one of the theories has sufficient data to explain the syndrome in a convincing manner. Regarding the psychogenic theory, he expresses the opinion that this field has not been as yet sufficiently explored.

\section{REFERENCIAS}

1. AlbeaUX-FERnet, M.; AlajouAnine, P. \& PAQUIN, R. - Les manifestations pathologiques de la période prémenstruelle. Sem. Hôp. Paris, 24:462-466, 1945. 2. AMARAL, C. - Alergoses hormonais em ginecologia. An. Brasil. Gin. 24:85-102, 1947. 3. APPLEBY, B. P. - A study of premenstrual tension. Brit. Med. J. 170:391-393, 1960. 4. ARVAY, A. \& MYIRI, I. - L'étiologie du syndrome prémenstruel. Gynéc. prat. 13:439-451, 1962. 5. BENEDEK, $T$. \& RUBENSTEIN, B. B. - The correlations between ovarian activity and psychodynamic processes: the ovulative phase. Psychosom. Med. 1:245-270, 1936. 6. BICKERS, w. - Premenstrual tension and its relationship to water metabolism. Am. J. Obst. Gynec. 64:587-590, 1952. 7. BICKERS, W. \& WOODS, M. - Premenstrual tension. Its relation to abnormal water storage. New Engl. J. Med. 245:453-456, 1951. 8. BISKINS, M. S. - Nutritional deficiency in the etiology of menorrhagia, metrorrhagia, cystic mastitis and premenstrual tension; treatment with $B$ complex. J. Clin. Endocr. 3:227-234, 1943. 9. BRUSH, A. L. - Attitudes, emotional and physical symptoms commonly associated with menstruation in 100 women. Am. J. Ortho= psychiat. 8:286-301, 1938. 10. CAZZOLA, D. - Alcuni casi di tensioni premenstruale trattati con terapia desensibilizzante ed anti-istaminici. Rev. Obst. e Ginec. 9:509-543, 1954. 11. CHADWICK, M. - The psychological effects of menstruation. J. Nerv. \& Ment. Dis. 76:532-533, 1932. 12. COOKE, W. R. - The differential psychology of the American woman. Am. J. Obst. Gynec. 49:457-472, 1945. 13. DAY, L. A. - Cit. por Freed \& Kroger. ${ }^{18}$ 14. EICHNER, \& WALTNER, C. - Premenstrual tension. Med. Times, 83:711-779, 1955. 15. FORTIN, J. N. \& WITTKOWER, E. D. - Le syndrome de la tension pré-menstruelle en regard de la médicine psychosomatique. L'Un. Méd. Can. 89:859-865, 1960. 16. FORTIN, J. N.; WITTKOWER, E. D. \& KALTZ, F. - A psychosomatic approach to the premenstrual tension syndrome: a preliminary report. Can. Med. Ass. J. 79:978-981, 1958. 17. FRANK, R. T. - The hormonal causes of premenstrual tension. Arch. Neurol. a. Psychiat. 26:1053-1057, 1931. 18. FREED, S. C. \& KROGER, W. S. Psychologic manifestations of the menstrual cycle. Psychos. Med. 12:229-235, 1950. 19. GILLMAN, J. - The nature of the subjective reactions evoked in women by progesterone with especial reference to the problem of premenstrual tension. $J$. Clin. Endocrin., 2:157-160, 1942. 20. GOLUB, L. J. \& MENDUKE, H. - Teen-age dysmenorrhea and social adjustment. Am. J. Obst. Gynec. 85:433-436, 1963.21. GREENBLATT, R. B. - Syndrome of major menstrual molimina with hypermenorrhea alleviated by testosterone propionate. J.A.M.A., 115:120-121, 1940. 22. GREENE, R. \& DALTON, K. - The premenstrual syndrome. Brit. Med. J. 1:10071014, 1953. 23. HENRIKSEN, E. - The melancholies of menstruation, or premenstrual tension. Clin. Obst. Gynec. 5:252-259, 1962. 24. HORNEY, K. - Cit. por Freed \& Kroger. ${ }^{18}$ 25. ISRAEL, S. L. - Premenstrual tension. J.A.M.A. 
110:1721-1723, 1938, 26. KRASOWSKA, J. - The psychotic syndromes of premenstrual tension at the age of puberty. Ann Medicopsychol. 118:849-876, 1960. 27. LAMB, W. M.; ULETT, G. A.; MASTERS, W. H. \& ROBINSON, D. W. - Premenstrual tension: EEG, hormonal and psychiatric evaluation. Am. J. Psych. 109: 840-848, 1953. 28. LAMSON, E. T.; ELMADJIAN, F.; HOPE, J. M.; PINCUS, G. \& JORIAN, D. - Aldosterone excretion of normal, schizophrenic and psychoneurotic subjects. Cli. Endocr. Metab. 16:954, 1956. 29. MORTON, J. H. - Premenstrual tension. Am. J. Obst, Gynec. 60:343-352, 1950. 30. MORTON, J. H.; ADDITON, H.; ADDISON, R. G.; HUNT, L. \& SULLIVAN, J. J. - A clinical study of premenstrual tension. Am. J. Obst. Gynec. 65:1182-1191, 1953. 31. MUKHERJEE, C. - Premenstrual tension: a critical study of the syndrome. J. Ind. Med. Assoc. 24:81-97, 1954. 32. PAULSON, M. J. - Psychological concomitant of premenstrual tension. Am. J. Obst. Gynec. 81:733-738, 1961. 33. PELLANDA, E. B. - Estudo preliminar sôbre a etiologia da tensão pré-menstrual e suas relações com a hipertensão arterial e a eclâmpsia. Rev. Med. Rio Grande do Sul, 10:173-206, 1954. 34. PENNINGTON, V. M. - Meprobamate (Miltown) in premenstrual tension. J. Am. Med. Assoc. 164:638-640, 1957. 35. PUNDER, N. A. - Contribuição ao estudo da sindrome pré-menstrual. O Hospital, 62:857-864, 1962. 36. REES, L. - Psychosomatic aspects of the premenstrual tension syndrome. J. Mental Scien. 99: 62-73, 1953. 37. RIEBEL, F. A. - Allergic coryza at menstruation from ovarian hormone. Ann. Int. Med. 9:91-92, 1935. 38. ROBINSON, F. H., Jr. \& FARR, L. E. - The relation between clinical edema and excretion of an antidiuretic substance in the urine. Ann. Int. Med. 14:42-54, 1940. 39. ROGERS, J. - Menstrual disorders New Engl. J. Med. 270:356-359, 1964. 40. SHAINESS, N. - Psychiatric evaluation of premenstrual tension. New York State J. Med. 62:3573-3579, 1962. 41. SCHICK, A. - Premenstrual depression. Am. J. Psychother. 7:664-671, 1953. 42. STIEGLITZ, E. \& KIMBLE, S. - Premenstrual intoxication. Am. J. Ment. Scien. 218:616-623, 1949. 43. TAYLOR, H. C., Jr. - Vascular congestion and hyperemia. Their effect on function and structure in the female reproductive organs. Am. J. Obst. Gynec. 57:211-230 e 637-668, 1949. 44. THORN, G. H.; NELSON, K. R. \& THORN, D. W. - A study of the mechanism of edema associated with menstruation. Endocrinology, 22:155-163, 1938. 45. TORGHELE, J. R. - Premenstrual tension in psychotic women. J. Lancet, 77:163-170, 1957. 46. VEIT, H. - Psychosomatic aspects of premenstrual tension. Wisc. Med. J. 54:599-601, 1955. 47. ZONDEK, B. \& BROMBERG, J. M. - Cit. por Amaral ².

Departamento de Neuro-Psiquiatria-Psicologia - Faculdade de Medicina da U.M.G. - Belo Horizonte, MG - Brasil. 